\title{
Síndrome de Burnout em profissionais de Enfermagem no contexto da Atenção Básica
}

\author{
Burnout Syndrome in Nursing professionals in the context of Basic Care
}

Síndrome de Burnout en profesionales de Enfermería en el contexto de la Atención Básica

Júlia Fernanda da Silva ${ }^{1 *}$, Monique Cardoso Silveira ${ }^{1}$, Andréia Andrade dos Santos ${ }^{1}$, Márcio Antônio Resende ${ }^{1}$, Bianca Cristina Silva de Assis ${ }^{1}$.

\section{RESUMO}

Objetivo: Identificar estratégias para diminuir os riscos aos quais os profissionais da enfermagem da atenção básica estão expostos para o desenvolvimento da síndrome de Burnout. Revisão bibliográfica: A presente revisão narrativa busca, por meio de produções relevantes, analisar o contexto que leva ao aparecimento e ao desenvolvimento da Síndrome de Burnout em sujeitos que são determinantes para o cuidado na saúde: os profissionais de enfermagem. Além disso, entender melhor o processo saúde-doença desses indivíduos nos ambientes de trabalho que deveriam acolhê-los pode facilitar a compreensão das causas para o problema bem como de meios para evitá-lo e modos de resolver a questão, focando na prevenção e na promoção de saúde. Considerações finais: Fica clara a importância de se trazer à tona essa discussão de modo a melhorar a qualidade de vida dos profissionais de Enfermagem, e espera-se que esse estudo contribua para a construção acadêmica e humanizada desses indivíduos.

Palavras-chave: Profissionais de enfermagem, Ambiente de trabalho, Burnout.

\section{ABSTRACT}

Objective: Identify strategies to reduce the risks to which primary care nursing professionals are exposed to developing Burnout syndrome. Literature review: This narrative review seeks, through relevant productions, to analyze the context that leads to the emergence and development of Burnout Syndrome in subjects that are determinants for health care: nursing professionals. Moreover, better understanding the health-disease process of these individuals in the workplace that should shelter them can facilitate the understanding of the causes for the problem as well as the means to avoid it and ways to resolve the issue, focusing on health prevention and promotion. Final considerations: It is clear the importance of bringing up this discussion in order to improve the quality of life of nursing professionals, and it is expected that this study contributes to the academic and humanized construction of these individuals.

Key words: Nursing professionals, Work environment, Burnout.

\section{RESUMEN}

Objetivo: Identificar las estrategias para reducir los riesgos a los que los profesionales de enfermería de la atención primaria están expuestos al desarrollo del síndrome de Burnout. Revisión de literatura: Esta revisión narrativa busca, a través de producciones relevantes, analizar el contexto que conduce a la aparición y el desarrollo del Síndrome de Burnout en sujetos que son determinantes para la atención en la salud: los profesionales de enfermería. Además, comprender mejor el proceso de salud-enfermedad de estas personas en el lugar de trabajo que debería ampararlos puede facilitar la comprensión de las causas del problema, así como los medios para evitarlo y las formas de resolver la molestia, centrándose en la prevención y promoción

\footnotetext{
${ }^{1}$ Centro Universitário Presidente Tancredo de Almeida Neves - (UNIPTAN), São João del-Rei - MG.

*E-mail: juliafsilva02@hotmail.com
} 
de la salud. Consideraciones finales: Es evidente la importancia de plantear esta discusión para mejorar la calidad de vida de los profesionales de enfermería, y se espera que este estudio contribuya a la construcción académica y humanizada de estos individuos.

Palabras clave: Profesionales de enfermería, Ambiente de trabajo, Burnout.

\section{INTRODUÇÃO}

A equipe de enfermagem atuante na Atenção Primária depara-se cotidianamente com conflitos, dentre eles: familiares, vulnerabilidade social, violência urbana e rural que Ihes afeta em virtude das situações de trabalho e da peculiaridade de suas atividades. Dessa forma, torna-se essencial que esses indivíduos fiquem atentos à sua saúde física e mental (SIQUEIRA GFF, et al, 2013).

Além dos conflitos existente, há estressores ocupacionais que são vivenciados pela equipe no campo de trabalho e que afetam diretamente o seu bem-estar, como lidar com a dor e a angústia do paciente, mortes e perdas, elevada demanda de atendimentos, carga horária excessiva, condições desfavoráveis de trabalho, renumeração baixa e pouco reconhecimento profissional, entre outros fatores de riscos (AVELLAR LZ, et al, 2007; BORGES LO, et al, 2006).

A Síndrome de Burnout (SB) ou Síndrome do Esgotamento Profissional é um tipo de estresse ocupacional que pode acometer os profissionais. Caracteriza-se por uma doença cujo principal fator desencadeador de sintomas é o excesso de trabalho atrelado à pressão e às responsabilidades contínuas. Os profissionais acometidos por essa síndrome apresentam cansaço excessivo e estresse prolongado, desgastes esses que prejudicam os aspectos físicos e emocionais do indivíduo, podendo alcançar o esgotamento profissional. (KESTENBERG KV, 2018).

Segundo Monteiro L (2019), a SB é qualificado como uma síndrome ocupacional, que ocorre quando o trabalhador se encontra esgotado, sendo decorrente de determinadas situações vividas no ambiente profissional. Sabendo disso, é possível afirmar, de acordo com pesquisas estatísticas da International Stree Management (ISMA) que a prevalência desse transtorno no Brasil é elevada, já que se estima que $32 \%$ entre todos os trabalhadores brasileiros desenvolvam a SB. Ainda de acordo com as pesquisas elaboradas pela Isma-BR, entre os oito países que mais apresentam colaboradores com sintomas do Burnout, o Brasil está em segundo lugar, ficando apenas abaixo do Japão, onde $70 \%$ da população sofrem dessa síndrome.

Os profissionais afetados pela SB geralmente possuem uma rotina de trabalho exaustiva e estão sempre sob muita pressão e, por se esforçarem demais para atender às demandas impostas, esquecem-se de viver suas vidas pessoais com o descanso e o relaxamento de que todo mundo precisa. Esses indivíduos ficam em total alerta e, por estarem em ambientes onde a exigência é exagerada e por não saberem, ainda, impor limites, tornam-se profissionais perfeccionistas ao ponto de não aceitarem errar e cobrarem muito de si mesmos. Todo esse desconforto causa desiquilibro entre a vida profissional e a vida pessoal, que, muitas vezes, torna-se inexistente, já que o trabalhador não sabe mais separar as duas coisas (KESTENBERG KV, 2018).

Segunda a Organização Pan-americana de Saúde (OPAS) (2018), para identificar se o profissional está com a SB, torna-se necessário ficar atento aos seguintes sinais que já apontam o início da doença: nervosismo, aflições psicológicas, problemas físicos constantes, tais como dor de barriga, cansaço excessivo e tonturas, e vontade de ficar apenas em casa.

É considerável dizer que os profissionais da saúde, nesse caso os enfermeiros e as enfermeiras que trabalham na atenção básica, presenciam a falta de recursos e a desigualdade de uma forma bem próxima, uma vez que vidas podem ser colocadas em risco em virtude de haver descaso com os grupos sociais que não possuem os recursos necessários para conseguirem o acesso à saúde de que tanto necessitam e que precisam recorrer ao sistema público de saúde. Ao serem telespectadores dessa situação, esses profissionais 
tornam-se humanamente propensos a desenvolverem quadros de estresse, por não poderem ajudar essa grande quantidade de pessoas que procuram a atenção básica de saúde (OPAS, 2018).

Em maio de 2019, a Organização Mundial da Saúde (OMS) publicou a $11^{a}$ Revisão da Classificação Internacional de Doenças, na qual, pela primeira vez, a SB foi listada como um fenômeno ocupacional, sendo conceituada no documento como uma síndrome resultante do estresse crônico em um local de trabalho que não foi gerenciado com sucesso. Ressalta-se que a síndrome não é considerada uma doença e que é decorrente apenas de problemas relacionados à área profissional dos indivíduos. Para finalizar, a OMS ressaltou que, a partir desse reconhecimento oficial sobre a definição e os sintomas do Burnout, a organização iniciará operações com instruções baseadas em evidências sobre o bem-estar mental no local de trabalho a fim de remediar e, futuramente, prevenir a alta prevalência do transtorno pelo mundo (NAÇÕES UNIDAS, 2019).

Segundo o Ministério da Previdência Social, em 2007, foram afastados do trabalho 4,2 milhões de indivíduos, sendo que 3.852 destes foram diagnosticados com SB, contribuindo com implicações financeiras negativas, pois a síndrome tem sido associada a aposentadorias precoces, absenteísmo e rotatividade de trabalhadores (SEGURA O, 2017).

Segundo Cimiotti JP e Aiken LH (2011), os profissionais da enfermagem são mais susceptíveis ao desenvolvimento de estresse ocupacional relacionado à assistência aos pacientes por acompanharem seus pacientes cotidianamente, o que resulta em um processo gradual de desgaste e desmotivação.

Sendo assim, por se tratar de um tema atual, que ainda está ganhando visibilidade e estudos oficiais, a importância desse estudo está no potencial de auxiliar os profissionais a identificarem os sintomas e seguirem as diretrizes de tratamento e prevenção a fim de tornar cada vez menores as estatísticas de trabalhadores que sofrem com a SB.

O presente estudo tem como objetivo revisar na literatura e identificar as estratégias que bem sirvam aos profissionais da área da saúde em atenção básica, o quanto antes, para diminuir as chances de evolução da síndrome e os riscos aos quais os profissionais estão expostos para o desenvolvimento da SB.

\section{REVISÃO BIBLIOGRÁFICA}

\section{Significado do termo Síndrome de Burnout}

O termo Burnout foi criado em 1974 por Freudenberger, um psicanalista que estava vivenciando sentimento de fracasso na profissão, exaustão causada por excessivo desgaste de energia, irritabilidade, depressão, aborrecimento, dentre outros problemas, ou seja, uma síndrome que tinha como sintomatologia o esgotamento profissional. Esse termo deriva do verbo inglês to burn out, que tem como significado, em língua portuguesa, "queimar por completo", "esgotar-se" ou "desgastar-se" (tradução das autoras).

A SB pode ser definida como um conjunto de sinais e sintomas psicológicos que surge quando um trabalhador está inserido em um ambiente profissional com clima estressante e com alta carga de tensão, refletindo no colaborador os sinais de exaustão profissional (ANDRADE PS, CARDOSO TAO, 2012). O problema pode chegar ao extremo de deixar o profissional incapacitado para o trabalho, seja por sua saúde física, seja pela mente, seja por ambas.

\section{Características da Síndrome de Burnout}

Segundo Maslach C e Jackson SE (1981), a SB é avaliada segundo três características, discorridas a seguir. A primeira é a exaustão emocional, que consiste em redução ou falta de eficiência relacionada à exaustão emocional e à insatisfação por parte dos indivíduos, tanto no nível físico quanto no nível psicológico, em que o profissional, nesse caso o enfermeiro, não dispõe mais de energia e disposição para oferecer aos pacientes uma boa assistência. A segunda é a despersonalização, que é denominada como ausência de envolvimento por parte do colaborador nas funções relacionadas ao trabalho, por apresentar ansiedade, 
irritabilidade, desmotivação, falta de propósitos e falta de compromisso com a missão e a visão de trabalho. E a última é a redução da realização pessoal, caracterizada por baixa na sensação de eficiência em referência à sua ocupação e à sua vida pessoal; de modo que os profissionais tendem a fazer avaliação negativa de suas atitudes, afetando, assim, a efetuação do trabalho e a assistência aos pacientes, o que interfere em suas percepções de crescimento e realização pessoal.

Em casos mais avançados, o corpo e a mente podem apresentar sintomas mais graves, tais como: cansaço excessivo tanto físico quanto mental, dores contínuas de cabeça, alterações no apetite, insônia, falta de concentração, sentimentos emocionais de fracasso, insegurança, falta de otimismo, isolamento, fadiga, pressão alta, dores musculares, entre outros indícios, que podem tornar evidentes o declínio na qualidade de vida do indivíduo, tanto no âmbito profissional, afetando sua produtividade, quanto na área pessoal, tirando sua perspectiva de melhora e muitas vezes a própria vontade de se superar por si só (GIL-MONTE P, PEIRÓ JM, 2003).

Assim, tem-se profissionais que não conseguem sair do ciclo que leva à SB e que, além de se prejudicarem com isso, deixam a assistência básica ainda com mais precarização na sua ausência. Logo, tem-se um sistema opressivo que afeta a vida de sujeitos responsáveis pelo cuidado em saúde e os torna incapazes de praticar o acolhimento, a atenção e o cuidado universal, integral e equitativo preconizado pelo SUS. Então, tem-se que o próprio sistema que preconiza tais ferramentas não as utiliza com seus próprios funcionários, causando questões que inviabilizam a construção da saúde de modo universal e integral.

De acordo com Lipp MEN e Malagris LEN (2001), um dos maiores contratempos que os indivíduos sofrem em suas rotinas é o estresse, que, definido como uma condição do organismo humano, leva os profissionais a sentirem fortes tensão e esforço. Os danos que se desencadeiam a partir desse desequilíbrio podem acarretar doenças graves.

Esse estresse se encontra presente na maior parte dos indivíduos, e suas raízes podem estar ou não ligadas às suas vidas profissionais. Porém, é muito mais comum que a exaustão profissional se torne mais evidente no trabalho, levando o nome de esgotamento profissional e causando ineficiência profissional, quando o indivíduo começa a apresentar os sintomas da SB (CARLOTTO MS, PALAZZO LS, 2006; CARLOTTO MS, CÂMARA SG, 2004).

Como sugestão, Lunardi L, et al. (2011) reforça a importância de diálogo entre os profissionais da enfermagem com seus chefes, buscando estratégias para aliviarem as tensões, frustrações, e que os últimos estimulem a motivação dos primeiros por meio de educação continuada, grupos de reflexões, entre outras atividades. É preciso que a comunicação esteja aberta para que os enfermeiros tenham liberdade para discutir suas demandas a fim de poder buscar, junto com os seus superiores, formas de contornar o problema.

Essa motivação pode ser classificada de duas formas, conforme Herberg (MONOGRAFIAS BRASIL ESCOLA): a primeira é a forma intrínseca, caracterizada como "automotivação", em que o sujeito busca dentro de si mesmos motivos que ajudem a estimular a realização de alguma coisa; e a segunda forma é a extrínseca, que se caracteriza pelo estímulo vindo de outras pessoas.

As intervenções e as medidas preventivas devem ser implementadas de acordo com a carestia de cada profissional, levando em consideração que a solução da síndrome não depende apenas de um único fator a ser vencido, e sim de recursos multifatoriais. Algumas propostas para intervenções dos pacientes já acometidos e para prevenções da síndrome foram classificadas para melhor compreensão, sendo elas estratégias organizacionais, estratégias individuais e estratégias combinadas.

As estratégias organizacionais são medidas que buscam modificar o âmbito organizacional. São situações nas quais se desenvolvem as atividades, como o ambiente e o clima do trabalho (GARROSA HE, et al, 2002). Essas estratégias, como mudanças nos espaços físicos, melhoras nas condições de trabalho tanto materiais quanto de recursos humanos, além do reconhecimento profissional, ajudam na efetividade organizacional e reforçam as condições de trabalho. Segundo os autores Winnubst JAM e Schabracq MJ (1996), a estrutura organizacional é elemento central na formação da cultura e do clima no ambiente de trabalho e está 
intimamente ligada ao suporte social e à percepção que as pessoas têm dessa estrutura, pois é considerada uma variável essencial para qualquer proposta de intervenção em relação à Síndrome de Burnout, uma vez que, por meio do ambiente, é possível agir sobre as disfunções provenientes do modelo estrutural prevalente na organização.

Ainda, tem-se as estratégias individuais, já que, de acordo com Campos RG (2005), mesmo com a exaustão do profissional pelas condições do trabalho, ele ignora sua saúde mental e seu bem-estar, além dos meios disponíveis para sua comodidade e satisfação em saúde. Existem estratégias que o profissional pode utilizar para a prevenção da SB como estratégias de coping, que são esforços cognitivos e comportamentais utilizados pelo sujeito para se adaptar às situações adversas, auxiliando-o a lidar com situações estressantes do cotidiano para superar as dificuldades (RODRIGUES AB, CHAVES EC, 2006). Alguns autores reforçam a importância de adquirir hábitos de vida saudáveis para a manutenção do equilíbrio físico e mental. Essas mudanças, como prática de exercícios físicos, alimentação equilibrada, dormir bem e ter momentos de lazer, ajudam a diminuir os efeitos do estresse profissional (MURTA SG, TRÓCCOLI BT, 2007).

Por fim, há as estratégias combinadas, que definem que, além de mudanças no ambiente do trabalho e no indivíduo, é importante que aconteçam atividades preventivas e de promoção para análise e considerações dos problemas. Sugerem-se momentos de sensibilização e reflexões, como grupos, rodas de conversas que orientem os profissionais quanto aos riscos a que estão notórios e a identificação dos sintomas da síndrome (CARLOTTO MS, PALAZZO LS, 2006). Essas são estratégias coletivas para alcançarem o equilíbrio psíquico frente aos riscos do ambiente do trabalho e permitem a descrição das vivências dos trabalhadores quanto a experiências cotidianas, sentimentos, ansiedade, contribuindo assim, para a criação de vínculo e ajuda a minimizar o sofrimento (FERNANDES JD, et al, 2006).

Essas ações são expressivas a partir do momento em que permitem a integração da equipe, a troca de experiência e apoio mútuo, além de ajudarem na redução da tensão no ambiente de trabalho. As ações conjuntas, envolvendo o profissional e a organização, são importantes na prevenção da SB, pois envolvem a participação de todos nas mudanças no contexto do trabalho (CARLOTTO MS, PALAZZO LS, 2006). Quando ocorre a união de toda a equipe, além do que foi apontado, encontra-se um ambiente favorável à conversa, ao entendimento e a melhor adaptação, de maneira que os enfermeiros possam se sentir amparados e mais alertas aos sinais da SB a fim de não chegar aos extremos do problema. Dessa forma, ganham não só a equipe de enfermagem, mas a unidade básica de saúde e, principalmente, os pacientes que chegam ao local buscando ajuda.

\section{A Síndrome de Burnout nos profissionais de Enfermagem}

Para mais, baseando-se em Martins LF, et al (2014) e em suas pesquisas com diversos autores, é possível inferir que os profissionais da área da saúde, principalmente no Brasil, estão mais sujeitos ao desenvolvimento da SB. Isso ocorre devido à formatação da Atenção Primária à Saúde (APS) no Sistema Único de Saúde (SUS), que, por meio da Estratégia de Saúde da Família (ESF), coloca esses indivíduos no papel central não apenas de tratamentos, mas também de programas de prevenção de doenças e promoção de saúde bem como acolhimento, cuidado e acompanhamento dos pacientes, de suas famílias e da comunidade. Assim, buscando a integralidade do atendimento enquanto princípio do SUS, o agente de saúde lida muito proximamente com cada demanda individual e comunitária e fica exposto a estressores psicossociais, tais como relacionamento interpessoal no trabalho.

Ainda, de acordo com Azevedo BDS, et al. (2017), o serviço de enfermagem, abrangendo técnicos, auxiliares e enfermeiros, está sujeito à baixa qualidade de vida no trabalho (QVT), principalmente no que concerne os últimos. Os trabalhadores dessa área percebem grandes demandas psicológicas e baixo controle sobre suas atuações somadas à grande responsabilidade que o ofício demanda. Por estarem responsáveis pela coordenação do funcionamento da ESF, lidarem diretamente com o público todos os dias o dia todo e ainda terem de responder a médicos e a gestões superiores, os enfermeiros se deparam com enormes 
responsabilidades diariamente. Ademais, muitas vezes, são influenciados pelas inquietações dos pacientes, uma vez que conhecem todo o processo de saúde e doença destes, bem como toda a comunidade e o funcionamento, as violências e as vulnerabilidades a que ela está sujeita. Tamanho convívio denota familiaridade que, por vezes, torna-se excessiva, no sentido de interferir na vivência profissional - e consequentemente pessoal - da equipe de enfermagem.

Dessa forma, pode-se conotar que, dentre os profissionais da área da saúde, os enfermeiros estejam mais sujeitos à SB, uma vez que se ocupam mais de perto dos pacientes, principalmente na APS, ao atenderem e acompanharem a comunidade diariamente (o que, na maioria das vezes, não se aplica aos médicos, por exemplo). Em estudo, Silva CCS, et al. (2017) corroboram essa afirmação, considerando as hierarquias e os conflitos presentes entre esses profissionais, além dos perigos e das insalubridades inerentes à profissão. Ademais, os autores consideram a carga de trabalho e a complexidade das tarefas exigidas dos enfermeiros como acima daquelas suportadas por estes, bem como analisam a falta de reconhecimento da função como um fator estressor.

Para mais, além da independência e da interdependência da equipe na APS - com as atividades reais dos enfermeiros sendo multidirecionada entre indivíduo, família e comunidade, há o viés de liderança ocupado por esses profissionais, que implica coordenar equipe, consultar e realizar exames, visitar domicílios e elaborar ações de prevenção e promoção de saúde para todos os sujeitos do território circunscrito. Ademais, a falta de recursos desde humanos até materiais, a baixa remuneração e o consequente acúmulo de mais de um trabalho como propiciadores da SB (LORENZ VR, GUIRARDDELLO EB, 2014).

Portanto, cabe ressaltar que o serviço de enfermagem precisa de um olhar mais atento no que tange à sua saúde para evitar que se chegue às altas demandas, às altas cobranças e, consequentemente, à SB. Assim, o cuidado com os trabalhadores deve ser prioridade, o que pode ser feito por meio de atividades físicas, por exemplo. Marziale MHP e Garcia GPA (2018) apontam que essa intervenção, além de poder ajudar a diminuir o estresse, pode melhorar desde a concentração e a agilidade mental até a satisfação no ambiente de trabalho, com melhores relacionamentos entre colegas, menor absenteísmo e redução de acidentes de trabalho.

\section{CONSIDERAÇÕES FINAIS}

Verificou-se nesse trabalho a relevância do estudo sobre Burnout e os principais fatores de risco que desencadeiam a síndrome, assim como a importância de propor medidas para extirpação ou minimização das consequências desse distúrbio. Durante o estudo, percebeu-se que pesquisas relacionadas ao tema são ainda escassas, mesmo sendo um assunto que deveria ser analisado com um olhar mais atencioso, pois afeta não somente os profissionais de saúde, mas também os usuários que recebem seus cuidados. Percebeu-se, ainda, a maior correlação da síndrome com o serviço de enfermagem, sendo necessário, portanto, rever e reorganizar o sistema da APS a fim de que esses profissionais não sejam sobrecarregados em sua lida direta e constante com as altas demandas comunitárias.

\section{REFERÊNCIAS}

1. ANDRADE PS, CARDOSO TAO. Prazer e dor na docência: revisão bibliográfica sobre a Síndrome de Burnout. Saúde e Sociedade, 2012; 21(1): 129-140.

2. AVELAR LZ, et al. Sofrimento psíquico em trabalhadores de enfermagem de uma unidade de oncologia. Psicologia em Estudo, 2007; 12(3): 475-481.

3. AZEVEDO BDS, et al. Estresse ocupacional e insatisfação com a qualidade de vida no trabalho da enfermagem. Texto \& Contexto Enfermagem, 2017; 26(1): 1-11.

4. BORGES LO. Os valores organizacionais e a Síndrome de Burnout: Dois momentos em uma maternidade pública. Psicologia: Reflexão e Crítica, 2006; 19(1): 34-43.

5. CAMPOS RG. Burnout: uma revisão integrativa na enfermagem oncológica [dissertação de mestrado]. Ribeirão Preto (SP): Universidade de São Paulo, 2005. 
6. CARLOTTO MS, CÂMARA SG. Análise fatorial do Malasch Burnout Inventory (MBI) em uma amostra de professores de instituições particulares. Psicologia em Estudo, 2004; 9(3): 499-505.

7. CARLOTTO MS, PALAZZO LS. Síndrome de Burnout e fatores associados: um estudo epidemiológico com professores. Cadernos de Saúde Pública, 2006; 22(5):1017-1026.

8. CIMIOTTI JP, AIKEN LH. Burnout - Gestão em Enfermagem: ferramentas para a prática segura. São Caetano do Sul, SP: Yendis, 2011.

9. FERNANDES JD, et al. Saúde mental e trabalho: significados e limites de modelos teóricos. Rev Latino-Am Enfermagem, 2006; 14(5): 803-811

10. GARROSA HE, et al. Prevenção e intervenção na síndrome de Burnout: como prevenir (ou remediar) o processo de Burnout. In: BENEVIDES PAMT, (Org.). Burnout: quando o trabalho ameaça o bem-estar do trabalhador. São Paulo: Editora Casa do Psicólogo; 2002, p.224-267.

11. GIL-MONTE P, PEIRÓ JM. Desgaste psíquico en el trabajo: el síndrome de quemarse. Madrid: Síntesis, 1997.

12. ISMA - International Stress Management Association. Estocolmo, Suécia: ISMA; 2003. (Bulletin).

13. KESTENBERG KV. Síndrome de Burnout: O que é, os sintomas e o tratamento. Psicologia viva [S. I.]: 18 ago. 2018. Disponível em: https://www.psicologiaviva.com.br/blog/sindrome-de-burnout/. Acesso em: 15 set. 2019.

14. LIPP MEN, MALAGRIS LEN. O stress emocional e seu tratamento. In RANGÉ B. (Org.), Psicoterapias cognitivocomportamentais: um diálogo com a psiquiatria. Porto Alegre: Artmed, 2001.

15. LORENZ VR, GUIRARDELLO EB. O ambiente da prática profissional e Burnout em enfermeiros na atenção básica. Rev. Latino-Am. Enfermagem, nov./dez. 2014; 22 (6): 926-933.

16. LUNARDI L, et.al. Sofrimento moral e a dimensão ética no trabalho da enfermagem. Revista Brasileira de Enfermagem, jul./ago., 2009; 62(4): 599-603.

17. MARTINS LF, et al. Esgotamento entre profissionais da Atenção Primária à Saúde. Ciência \& Saúde Coletiva, 2014; 19(12): 4939-4750.

18. MARZIALE MHP, GARCIA GPA. Indicadores de esgotamento profissional em trabalhadores da Atenção Primária à Saúde. Rev. Bras. Enferm. (internet), 2018; 78(supl. 5): 2469-2478.

19. MASLACH C, JACKSON SE. The measurement of experienced Burnout. Journal of Occupational Behavior, 1981; 2(2): 99-113.

20. MONOGRAFIAS BRASIL ESCOLA. Teoria dos dois fatores. [S.I.]. Disponível em: https://monografias.brasilescola.uol.com.br/administracao-financas/teoria-dos-dois-fatores.htm. Acesso em: 20 set. 2019.

21. MONTEIRO L. OMS classifica a Síndrome de Burnout como doença. [S.I.]: 31 maio 2019. Disponível em: https://super.abril.com.br/saude/oms-classifica-a-sindrome-de-burnout-como-doenca/. Acesso em: 23 ago. 2019.

22. MURTA SG, TRÓCCOLI BT. Stress ocupacional em bombeiros: efeitos de intervenção baseada em avaliação de necessidades. Estud Psicol, 2007; 24(1): 41-51.

23. NAÇÕES UNIDAS BRASIL. Síndrome de Burnout é detalhada em classificação da OMS. [S.I.]: 29 maio 2019. Disponível em: https://nacoesunidas.org/sindrome-de-burnout-e-detalhada-em-classificacao-internacional-da-oms/. Acesso em: 15 set. 2019.

24. OPAS - Organização Pan-Americana da Saúde. Ampliação do papel dos enfermeiros na atenção primária à saúde. Washington, D.C.: OPAS; 2018

25. RODRIGUES AB, CHAVES EC. Fatores estressantes e estratégias de coping dos enfermeiros atuantes em oncologia. Rev Latino-Am Enfermagem, jan.fev. 2018; 16(1): 24-28.

26. SEGURA O. Burnout: concepts and implications affecting public health. Biomedica, 2014; 34(4): 535-545

27. SILVA CCS, et al. Burnout e tecnologias em saúde no contexto da enfermagem na Atenção Primária à Saúde. Esc Anna Nery, 2017; 21 (2): e 20170031.

28. SIQUEIRA GFF, et al. Trabalho do Enfermeiro na atenção primaria em saúde: conhecimentos dos fatores estressores. Rev Ciênc Saúde Nova Esperança, 2013; 11(2): 72-85.

29. WINNUBST JAM, SCHABRACQ MJ. Social Support, Stress and Organization: Towards Optimal Matching. In: The Handbook of Work and Health Psychology. Manhattan: Wiley, 1996. 